\title{
Deep tissue near-infrared imaging for vascular network analysis
}

\author{
Kübra Seker* and Mehmet Engin \\ Department of Electrical \& Electronics Engineering \\ Faculty of Engineering \\ Ege University Bornova, Izmir, Turkey, 35100 \\ *kubraaseker@gmail.com
}

Received 22 March 2016

Accepted 30 August 2016

Published 6 October 2016

\begin{abstract}
Subcutaneous vein network plays important roles to maintain microcirculation that is related to some diagnostic aspects. Despite developments of optical imaging technologies, still the difficulties about deep skin vascular imaging have been continued. On the other hand, since hemoglobin concentration of human blood has key role in the veins imaging by optical manner, the used wavelength in vascular imaging, must be chosen considering absorption of hemoglobin. In this research, we constructed a near infrared (NIR) light source because of lower absorption of hemoglobin in this optical region. To obtain vascular image, reflectance geometry was used. Next, from recorded images, vascular network analysis, such as calculation of width of vascular of interest and complexity of selected region were implemented. By comparing with other modalities, we observed that proposed imaging system has great advantages including nonionized radiation, moderate penetration depth of $0.5-3 \mathrm{~mm}$ and diameter of $1 \mathrm{~mm}$, cost-effective and algorithmic simplicity for analysis.
\end{abstract}

Keywords: Vascular NIR imaging; manufacturing liquid and solid phantoms; diffuse optical imaging; image processing and analysis; optical imaging system design.

\section{Introduction}

Subcutaneous vein network is related to blood circulation therefore, it plays important roles to maintain human skin tissue. Despite developments of optical imaging technologies, still the difficulties about deep skin imaging have been continued. ${ }^{1,2}$ In recent years, many technologies have been introduced to solve subcutaneous veins imaging problems. ${ }^{3-6}$ Venographic guidance provides sufficient contrast by using special contrast agent for radiographically imaging. ${ }^{7}$ On the other hand, ultrasound-based imaging systems prevent patient against radiation but sophisticated training is required. ${ }^{8,9}$ The other technique named transilluminator allows visualization of

*Corresponding author.

This is an Open Access article published by World Scientific Publishing Company. It is distributed under the terms of the Creative Commons Attribution 4.0 (CC-BY) License. Further distribution of this work is permitted, provided the original work is properly cited. 
veins but its efficiency may be dependent on the size of body region. ${ }^{10,11}$ Therefore, diffuse optical techniques have evolved as a powerful tool to functionally substitute these expensive modalities in some specific diagnostic investigations. ${ }^{12}$

In the following, the state of art about near infrared (NIR) imaging is given shortly; In the medical diagnosis molecular imaging plays important roles in conjunction with sophisticated, high-resolution detection tasks. While many kinds of imaging modalities have been implemented for this aim, intravascular near infrared fluorescence imaging (NIRF) can provide high-resolution monitoring of human coronary artery Nowadays, animal studies have showed feasibility of NIRF for detecting inflammation and fibrin deposition in coronary-sized arteries. ${ }^{13}$

Near infrared fluorescence (NIRF) molecular imaging provides promise as a new medical imaging tool that has more sensitivity, but without the radioactivity. Today, NIRF modality has emerged in clinical conditions using indocyanine green (ICG) as a nontargeting NIRF contrast agent to demark the blood and lymphatic vasculator. ${ }^{14}$

On the other hand, in the literature, there is an experimental growth of the number of publications on functional near-infrared imaging (FNIRI). It is predictable that this progress will be sustained, because the potential of FNIRI is still far from being fully maturated.

As a result, in the near future, FNIRI imaging will provide an increasing spatial and temporal resolution. Therefore, it will enable other progress in signal or data analysis. In addition, FNIRI can be easily combined with further functional imaging modalities, such as; FMRI, EEG, PET and MEG.

And also, since FMRI can be miniaturized and made wearable, this provides new field of applications, such as therapy for severely impaired stroke patients. ${ }^{15}$

The other feasibility of NIR imaging is related to the real-time visualization of peripheral veins. Venipuncture is a medical progress of obtaining intravenous access. While a peripheral vein can be accessed in a single attempt, in generally, this attempt number are between 2 and 10 attempts. Excessive venipunctures are a significant question (time-and resource-consuming, and patient's disconfortability). To address this situation, nowadays, there are several medical devices that utilize NIR to help vascular access procedures. These commercial NIR imaging devices provide a valuable clinical function. ${ }^{16}$
Since hemoglobin concentration of blood has key role in the veins imaging by optical manner, the used wavelength in vascular imaging must be chosen considering absorption of hemoglobin. In this study, we constructed a NIR light source module to image in measurement circumstance. Reflectance geometry was used to obtain vascular structure. After recording of camera-based images, vascular network analysis such as calculation of width of vascular of interest and complexity of vascular region were implemented. By comparing with other modalities, ${ }^{8,17,18}$ proposed imaging system has great advantages including; nonionized radiation moderate penetration depth of $0.5-3 \mathrm{~mm}$ and diameter of $1 \mathrm{~mm}$, cost-effective and algorithmic simplicity for analysis of related vascular images.

\section{Methods}

\subsection{Vascular imaging}

Generally the structure of the vascular (vein) patterns can be identified and captured by infrared sensors. The visibility of the vascular pattern depends on some factors such as age, thickness of the skin, ambient temperature, physical activity, depth of the vascular region. ${ }^{19}$ In practice, there are two types of the imaging modalities for vascular imaging, namely far-infrared (FIR) and NIR methods. FIR modality has wavelength within the range $8-14 \mu \mathrm{m}$ but it is sensitive to ambient conditions and therefore does not provide a stable image quality. NIR imaging works within the range $700-1000 \mathrm{~nm}$ and it has good image quality. And also this band is more tolerant to changes in environmental and body conditions. ${ }^{19}$ In this imaging, LED or laser light source illuminates the subject's skin with NIR light. Coming light penetrates skin and subcutaneous fat layer effectively because of low absorption coefficient of this type of tissues. Then, some photons are absorbed or scattered in the forward direction by blood while they are scattered in all directions in skin and fat region. Consequently, blood appears as a dark region, whereas skin and fat appear lighter. Finally, the reflected photons produce an image in the camera. The recorded NIR image is processed and analyzed by computer. ${ }^{18}$ Before the measurement of vascular diameter (or thickness) and network complexity, it should be made NIR image quality analysis by some quantitative methods. 


\subsection{Experimental set-up}

The general setup of proposed optical system consists of an NIR LED-based illumination source, polarizers, infrared (IR) filter, a camera (Xenics Infrared Camera: The Xeva-1.7-320 unit is available with standard up to $1.7 \mu \mathrm{m}, \mathrm{InGaAs}$ detector arrays and comes in various speed versions: $60 \mathrm{~Hz}, 100 \mathrm{~Hz}$ and $350 \mathrm{~Hz}$.). LED light source was implemented for both disk and ring layout. Each LED module (disk/ring type) has 17 LED for $870 \mathrm{~nm}$ and $940 \mathrm{~nm}$, separately General schematic diagram of the NIR imaging system was given in Fig. 1.

In spectral window between $870 \mathrm{~nm}$ and $940 \mathrm{~nm}$, oxygenated and deoxygenated hemoglobin have same absorption coefficients approximately and water's absorption coefficient is very low for this optical region. This results are very useful to obtain optical penetration against overlapping (or suppressing) effects originated from water. Therefore, we used mentioned wavelengths for light source in our designed optical system. And also we observed that there is no difference between two designs (ring and disk shaped) regarding spot size and irradiation uniformity. Therefore, we only gave the imaging results in the case of ring shaped design for reporting.

In order to control LED powers, a current driver circuit was designed (Fig. 2). Since we have no laser speckle or computer tomography imaging systems in our laboratory, we aimed only phantom-based measurements to validate proposed optical system. In addition, to confirm the integrated sphere system measurements, a custom phantom with known characteristics is bought (INO Canada) and its optical coefficients are determined via integrated sphere system again for comparison. From that, it is observed negligible errors (differences) between both measurements.

\subsection{Phantom fabrication and characterization}

In biomedical optical imaging, the tissue mimic phantoms play important roles. The calibration of an optical imaging system requires, the use of repetitive measurements over phantom medium. For this aim, we fabricated a liquid phantom (for blood) and pork gelatin-based phantom (for skin tissue). The scattering properties were on the basis of the Mie theory. In our fabrication of liquid phantom; $0.1 \%$ intra lipid was selected as scattering material, while absorption was introduced with $0.2 \%$ amount of Indian ink. Then scattering and absorption materials were mixed with diluted water in the cylindrical tank. On the other hand, gelatin-based material was used to fabricate a solid phantom. In order to mimic scattering and absorbing properties of tissue to be imaged, Indian ink and lipid materials were added to base pork gelatin material. Since we plan only preliminarybased study, in that step we used mentioned materials (ink and lipids) for mimicking human (in-vivo) material. As a future study, we will plan clinically conducted experiments (under ethical board considerations). In this study, the proposed optical imaging system is also applied to human candidate as explained in Sec. 2.6 and we got

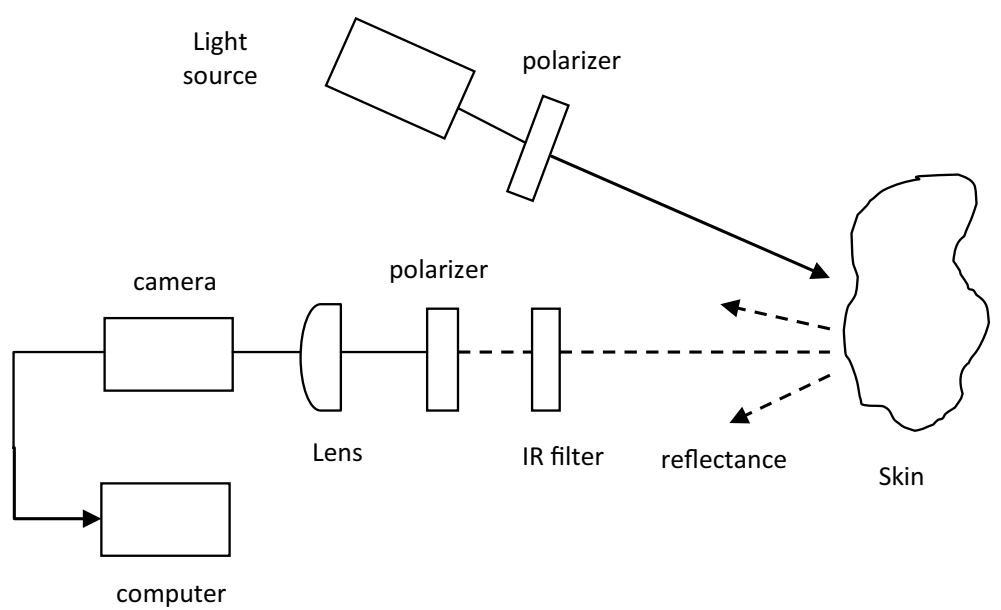

Fig. 1. Schematic diagram of NIR imaging system. 


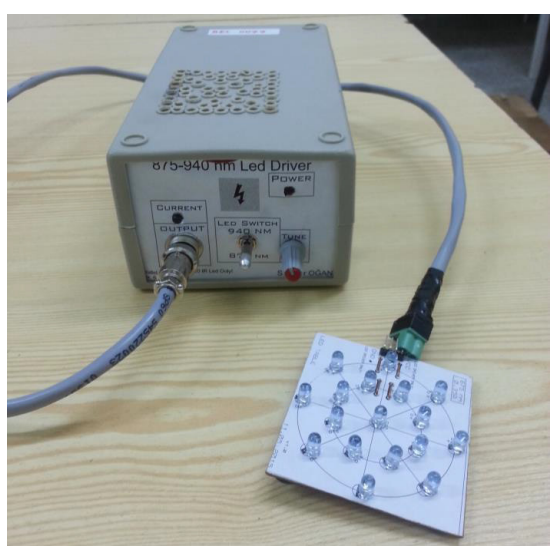

(a)

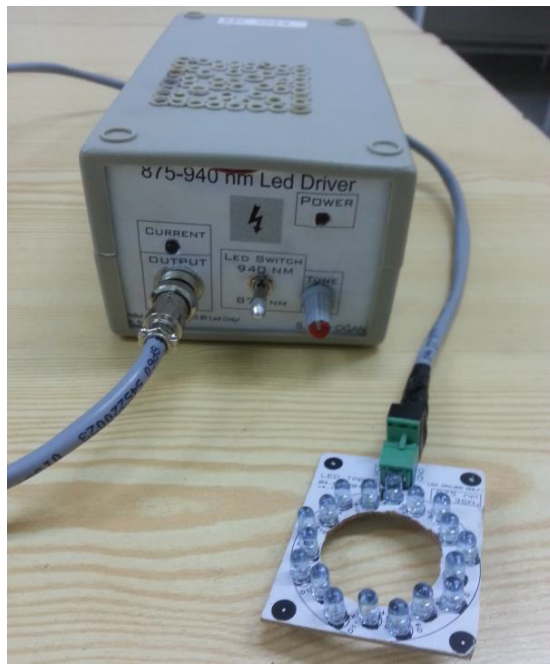

(b)

Fig. 2. LED light source device and LED layoults; (a) Disk shaped design and (b) Ring shaped design.

sufficient imaging performance from wrist and arm region. The details of phantoms fabrication process were given in literature. ${ }^{8}$ After fabrication process of liquid and solid (gelatin-gel) phantoms, their optical characterizations such as the absorption and scattering coefficients were measured by the inverse-adding-doubling (IAD) method. This method uses reflectance, transmittance and collimated transmittance measurements of the sample (phantom). ${ }^{20}$ In this method in order to obtain optical coefficients, iterative solving of the radiative transfer equation with the adding-doubling approach was used. In this calculation, the following assumptions were considered; the sample was to be an infinite planeparallel slab with given thickness and refractive index $(n)$, and the optical properties were to be uniform distributed in medium. However, the errors coming from measurement play critical role than the method itself. Consequently, proper measurements should be made more precisely in the IAD method. ${ }^{12}$ Given IAD method (algorithm) in Ref. 21, has a practical interface (calculator) for user. IAD calculation process needs the following input parameters; total (measured) reflectance, total (measured) transmittance, unscattered (measured collimated) transmittance, sample thickness $(\mathrm{mm})$, sample index of refraction. Then program calculates the following outputs (or results); absorption coefficients $\left(\mathrm{mm}^{-1}\right)$, scattering coefficient $\left(\mathrm{mm}^{-1}\right)$, average cosine of phase function, reduced scattered coefficient $\left(\mathrm{mm}^{-1}\right)$, iteration number.
The absorption coefficient $\left(\mu_{a}\right)$ describes the attenuation of the light depending on medium at a certain wavelength. In biological tissue, main absorbers are melanin and hemoglobin. The reduced scattering coefficient $\mu_{s}^{\prime}$, is related to the photon migration process in the tissues for diffuse regime. According to the following equation, scattering coefficient can be defined;

$$
\mu_{s}=\frac{\mu_{s}^{\prime}}{1-g} .
$$

The scattering coefficient $\mu_{s}$, describes the scattering properties of the tissue, where $g$ : denotes anisotropy factor. The IAD algorithm for reconstruction of optical properties, is shown in Fig. 3.

In order to use IAD; total reflection $R(\lambda)$, total transmission $T(\lambda)$ and collimated transmission $T_{\text {col }}(\lambda)$, refractive index $n$, and thickness $\mathrm{L}$, are obtained experimentally. In our experiments, for this aim, Ocean (USB4000) spectrometer and (ThorLabs IS36A-4) single integrating sphere system were used.

\section{(a) The transmission measurement}

The total transmission of sample, $T(\lambda)$ was calculated as follows:

$$
T(\lambda)=\frac{T_{s}-T_{\text {dark }}}{T_{\text {ref }}-T_{\text {dark }}},
$$

where $T_{s}, T_{\text {dark }}$ and $T_{\text {ref }}$ are spectra (from spectrometer) values for sample, dark condition and reference material (inner-wall), respectively when 


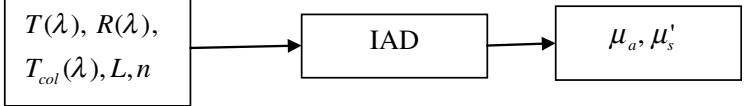

Fig. 3. IAD algorithm for retrival optical coefficients.

integrating sphere system was used in transmission configuration. Transmittance increases with the wavelength, and also it heavily depends on the phantom thickness.

(b) The total reflectance measurements $R(\lambda)$, total reflectance is defined as follows:

$$
R(\lambda)=\frac{\left(R_{s}-R_{\text {dark }}\right)}{\left(R_{\text {ref }}-R_{\text {dark }}\right)} \cdot r_{\text {std }}
$$

where $R_{s}, R_{\text {ref }}, R_{\text {dark }}$ are spectra values (from spectrometer) for sample and reference material (inner-wall) and dark condition, respectively when integrating sphere system was used in the reflectance configuration. The reflectivity efficiency of sphere inner surface was given by, $r_{\text {std }}$.

(c) The collimated transmittance measurement $T_{\text {col }}(\lambda)$ collimated or unscattered transmittance was measured using a collimator and was defined as transmittance calculation Eq. (2) However, the collimated transmittance measurements were mostly depending on low amounts of light, so a poor signal-to-noise ratio leads to inaccurate data (or signal).

Finally, the mentioned above three measurements values; $T(\lambda), R(\lambda)$ and $T_{\text {col }}(\lambda)$ and sample's refractive index and thickness were submitted to IAD algorithm which was developed. ${ }^{20,21}$ This method relies on the iterative solving the radiative transfer equations with the adding-doubling when the sample was assumed to be an infinite planeparallel slab with known thickness and refractive index, $n$.

\section{(d) The results of phantom measurements}

In order to mimic blood and skin tissue, we fabricated liquid and solid (pork gelatin) phantoms, respectively. First, we will report liquid phantoms optical measurements. The following figure in (Fig. 4) shows the acquired spectra from which, $T(\lambda), R(\lambda)$ and $T_{\text {col }}(\lambda)$ were calculated.

Finally, optical properties were calculated from IAD method by using above measured spectra at $875 \mathrm{~nm}$. The reduced scattered coefficient $\left(\mu_{s}^{\prime}\right)$ was obtained as $\quad<<8 \mathrm{~cm}^{-1}$ and absorption coefficient $\left(\mu_{a}\right)$ was as $0.2 \mathrm{~cm}^{-1}$. We observed that, these obtained values are matched closely to data of real human skin found in the literature. ${ }^{10}$

In the next step, the gelatin-gel phantom optical measurements were implemented in the same way. The related, $T(\lambda), R(\lambda)$ and $T_{\text {col }}(\lambda)$ factors were calculated. Obtained spectra examples were given in Fig. 5. In order to obtain scattering properties that are matched closely to real human skin, we added the additional lipid solution $(3 \mathrm{~mL})$ into the gelatin-gel solution $(5 \mathrm{~g})$.

In the same way; final optical coefficients were calculated by IAD at $875 \mathrm{~nm}\left(\mu_{s}^{\prime}=0.341 \mathrm{~cm}^{-1}\right.$, $\left.\mu_{a} \cong 4.21 \mathrm{~cm}^{-1}\right)$. We observed that these numerical values were different from that of literature. Potential errors sources for that situation, can be assumed as follows; (a) changing of gelatin characteristics and manufacturing conditions by time and, (b) using the inner wall of the sphere instead of specific reference material.

\subsection{Vascular network analysis}

In order to study on vascular imaging, we developed in-vitro medium by using previous designed gelatin phantom. To mimic vascular network, plastic tubes with diameters $1 \mathrm{~mm}$ and $3 \mathrm{~mm}$ were buried into skin phantom material for different measurements. Figure 6 shows gelatin phantom material with a plastic tube.

For this imaging, used optical imaging system (given in Fig. 1) consists of following same components: disc and/or ring shaped LED array modules, LED current driver circuit, polarizers, and CMOs NIR camera. Imaging process was repeated for wavelength $857 \mathrm{~nm}$ and $940 \mathrm{~nm}$, while diameter of plastic tubes were set to $1 \mathrm{~mm}$ and $3 \mathrm{~mm}$. For all measurements, the depth of tube was set to $3.5 \mathrm{~mm}$ from surface. While, superficial reflections were canceled by cross-polarization, the output power of LED modules was changed by current driver circuit for avoiding optical saturation condition (typical output power of driver circuits was set to $40 \mathrm{~mW}$ ). After recording of images, we have used following image processing and analysis process for enhancing and extracting of vessel structure and also calculating of diameters and vascular complexity parameter as flow chart given in Fig. 7. 


\section{K. Seker \& M. Engin}

\section{Enhancement and thresholding}

Images represented in the RGB color model consist of three components images, one component for each primary color. For simplicity on image processing, color model-based images were converted to the gray-level based images. In order to get enhanced or sharped image, we have used contrast limited adaptive histogram equalization (CLAHE). In detail, CLAHE algorithm enhances an image by small data regions called "tiles" rather than entire image. The resulting neighboring tiles are then stitched back seamlessly using bilinear interpolation. For example, in Ref. 22, CLAHE method is applied to myocardial perfusion images in color space. The related flowchart is given in Fig. $8 .^{22,23}$ However, this algorithm can amplify some noisy components during image enhancement. In order to control

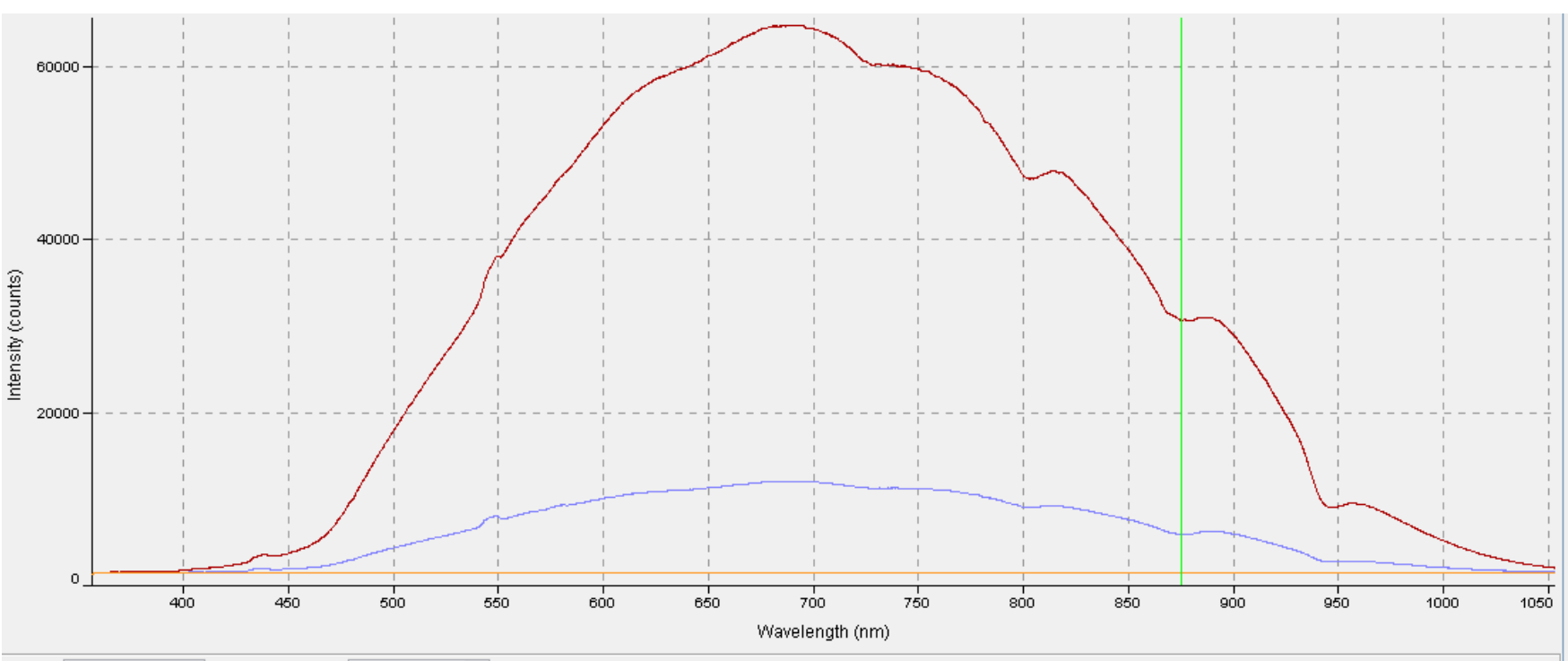

Source: USB 4F02261 $\checkmark$ Wavelength $(\mathrm{nm}): \quad 875,14 \div$

(a) The transmission based spectra $\left(T_{\mathrm{s}}\right.$ : middle trace, $T_{\text {dark }}$ : lower trace and $T_{\text {ref }}$ : upper trace).

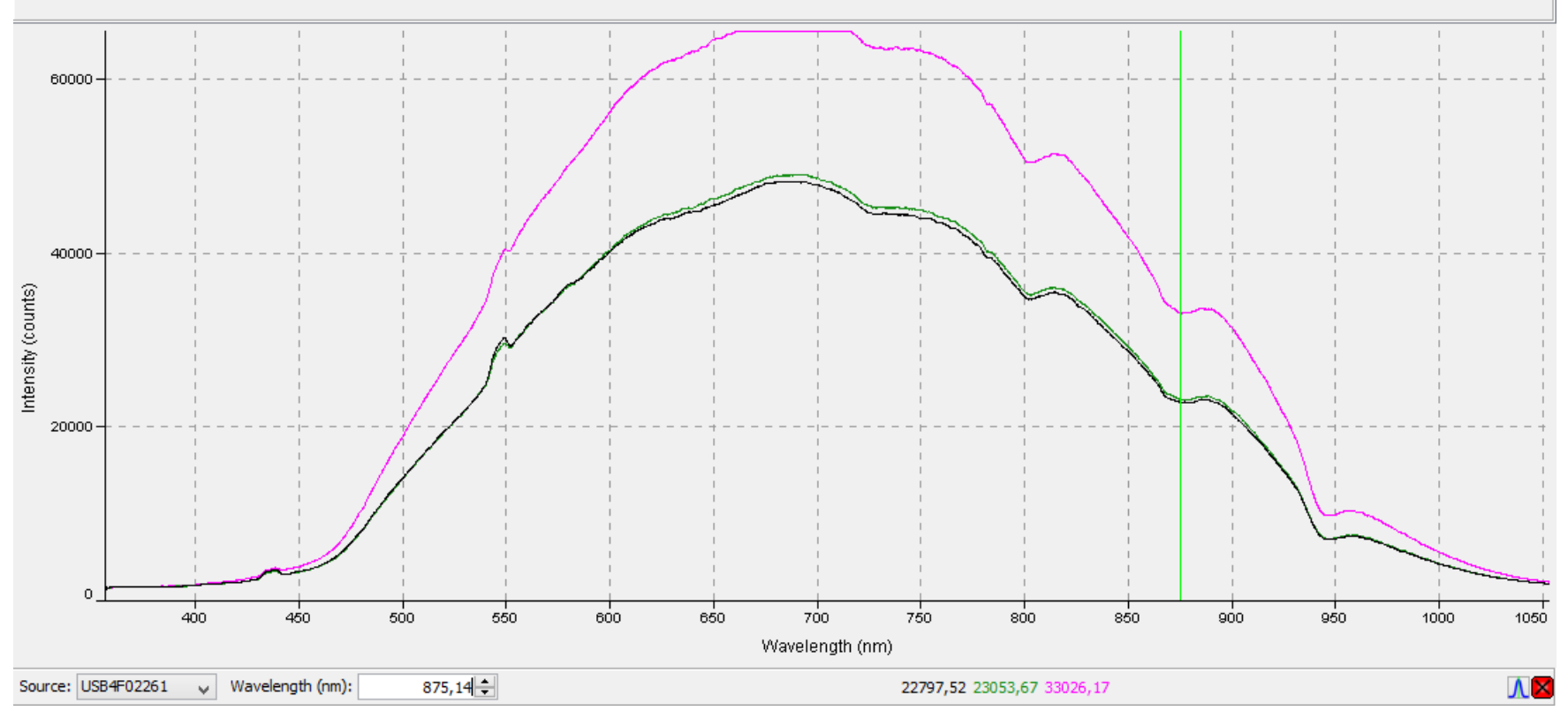

(b) The reflectance based spectra $\left(R_{s}\right.$ : middle trace, $R_{\text {ref }}$ : upper trace, $R_{\text {dark }}$ : lower trace).

Fig. 4. The measured liquid phantom spectra examples. 


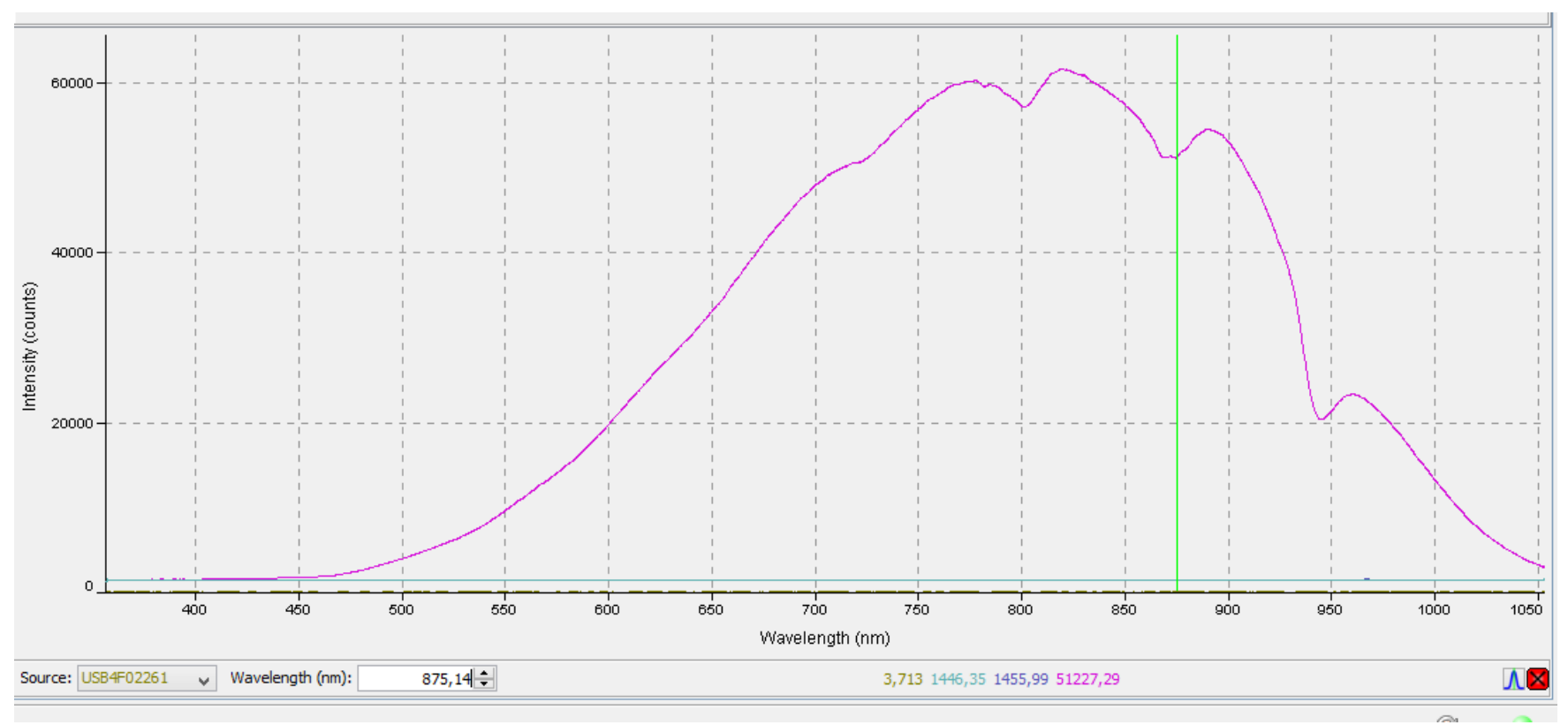

(c) The collimated transmission based spectra $\left(T_{s}^{\prime}\right.$ : middle trace, $T_{\text {dark }}^{\prime}$ : lower trace, and $T_{\text {ref }}^{\prime}$ : upper trace).

Fig. 4. (Continued)

noise level, entire image is divided into user defined sub-images whose size and clip-level are adaptively determined by CLAHE method.
In the thresholding, we used local entropy-based method that was released on the Co-occurrence matrix of image. In an image, the spatial information is joint gray-level values of the pixel to be

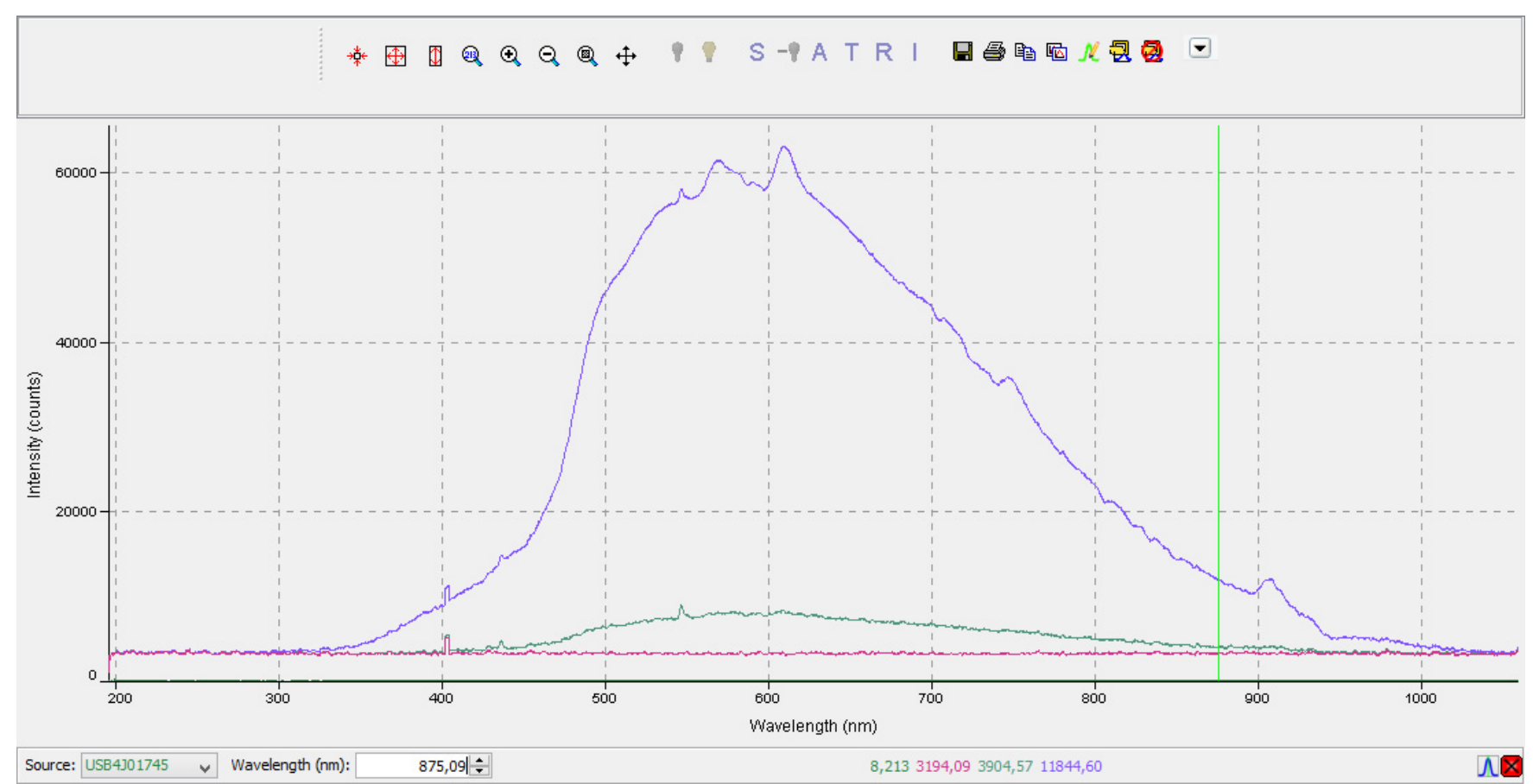

(a) The transmission based spectra $\left(T_{s}\right.$ : middle trace, $T_{\text {dark }}$ : lower trace and $T_{\text {ref }}$ : upper trace).

Fig. 5. The gelatin phantom spectra examples. 


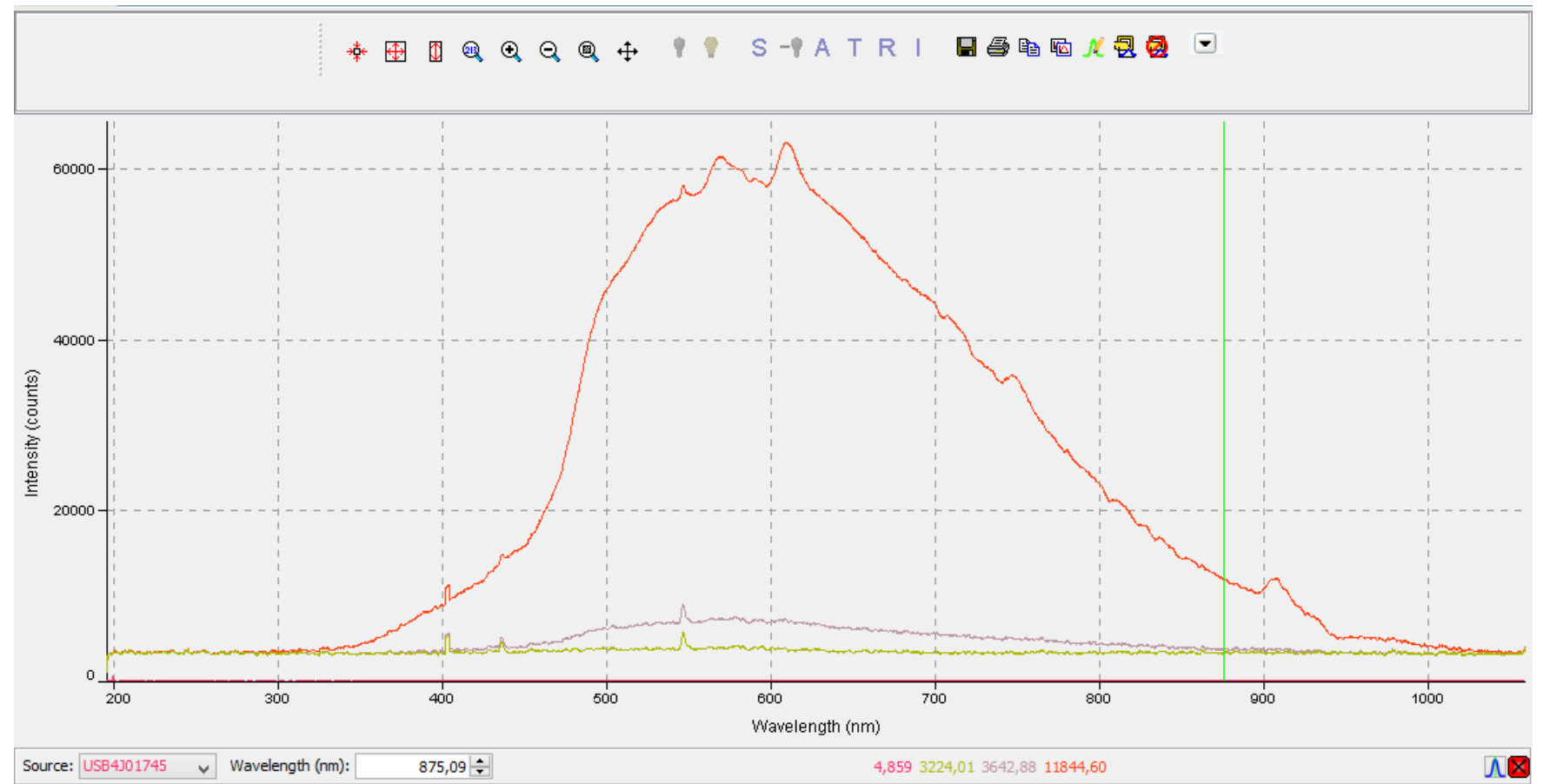

(b) The reflectance based spectra $\left(R_{s}\right.$ : middle trace, $R_{\text {ref }}$ : upper trace, $R_{\text {dark }}$ : lower trace).

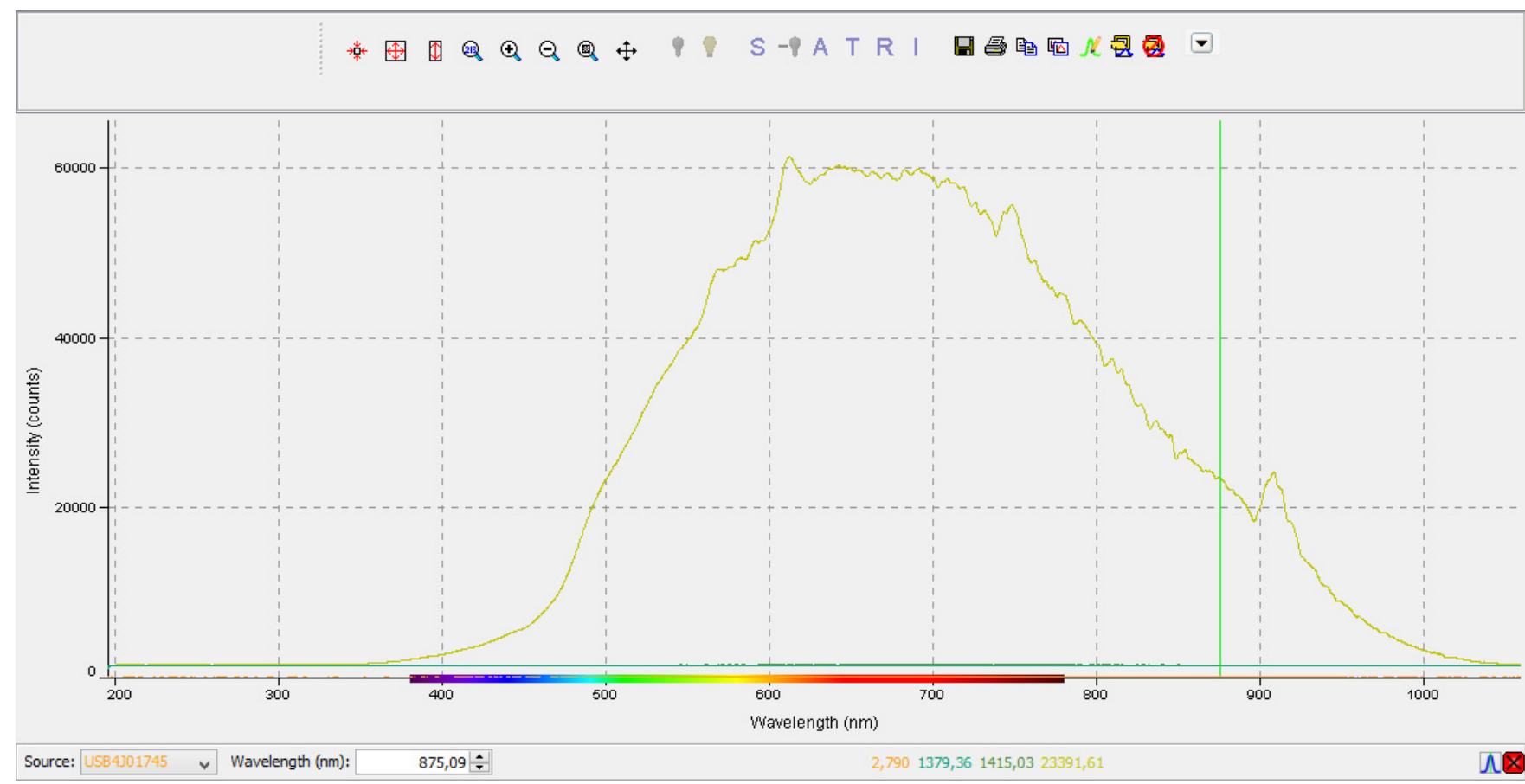

(c) The collimated transmission based spectra $\left(T_{s}^{\prime}\right.$ : middle trace, $T_{\text {dark }}^{\prime}$ : lower trace and $T_{\text {ref }}^{\prime}$ : upper trace).

Fig. 5. (Continued)

segmented and its neighboring pixels are related to the gray-level co-occurance matrix (GLCM). Firstly, the $2 \mathrm{D}$ local cross entropy is determined at the local quadrants of the GLCM. Then, the 2D local cross- entropy is used to obtain optimal threshold level by entropy minimizing. All details of this approach are given in Ref. 24. After threshold process, we obtained a binary-image for next analysis (calculation). 


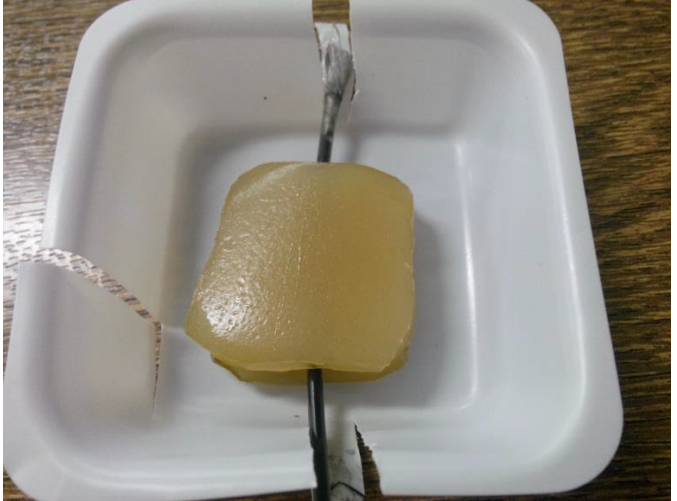

Fig. 6. The gelatin phantom with artificial vein.

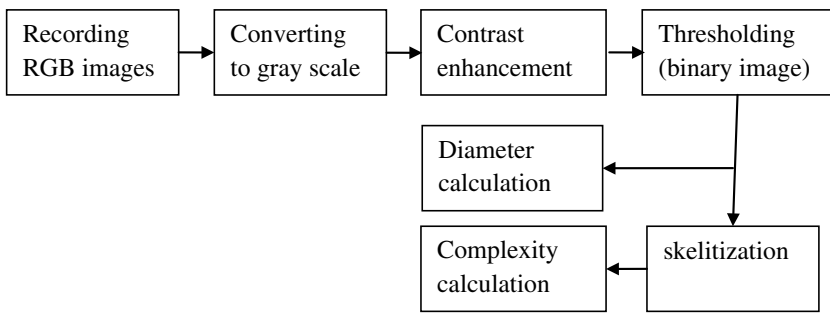

Fig. 7. Image processing and analysis flow diagram.

\section{Vessel diameter calculations}

The diameter or width of a vessel is defined as smallest distance between both surface of the vessel. For the measurement of this distance, the following mask (or matrix) was shifted over the vessel by vertically as in Fig. 8. Since vessel region was represented by dark ("0") pixels, for given any location, if masking result was set to 3 , then this point (A) can be considered as vessel surface.

By vertically shifting, when the second masking results was equal to 3 , so this location (B point) was taken as other vessel surface. In order to get real

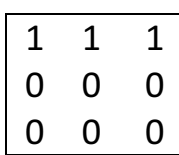

(a)

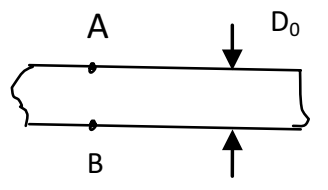

(b)
Fig. 9. Masking and shifting process; (a) Mask operator (b) Shifting of mask.

distance between $\mathrm{A}$ and $\mathrm{B}$ points, it should be required a scaling process for converting the pixel size to physical length ( $\mathrm{mm})$. For this aim: a scaling object whose size are known, is placed over the image. Finally, vessel diameter or width was obtained from the above mentioned description.

\section{Vessel complexity calculation}

The functional vascular density (FVD) is measure for representing geometrical complexity (or pattern complexity) of vascular structure at given binary image region. However, before calculating of FVD, the skelitization and thinning of foreground (vascular) is required. ${ }^{25}$ After iteratively implementation of both process, related vascular region width can be obtained as one pixel size (dark pixel, "0"). After that, FVD parameter is calculated over this image region. ${ }^{26}$

$$
\mathrm{FVD}=\frac{\sum_{i=0}^{N} \sum_{j=0}^{M} T(i, j)}{M \times N},
$$

where $T(i, j)$ represents vascular-based pixels ("0"), and $M \times N$ denotes size of full region of image.

\subsection{In-vitro imaging results}

By using own designed pork gelatin phantoms, to mimic human vascular model, different plastic tubes with two different diameters $(1 \mathrm{~mm}$ and $3 \mathrm{~mm})$ were

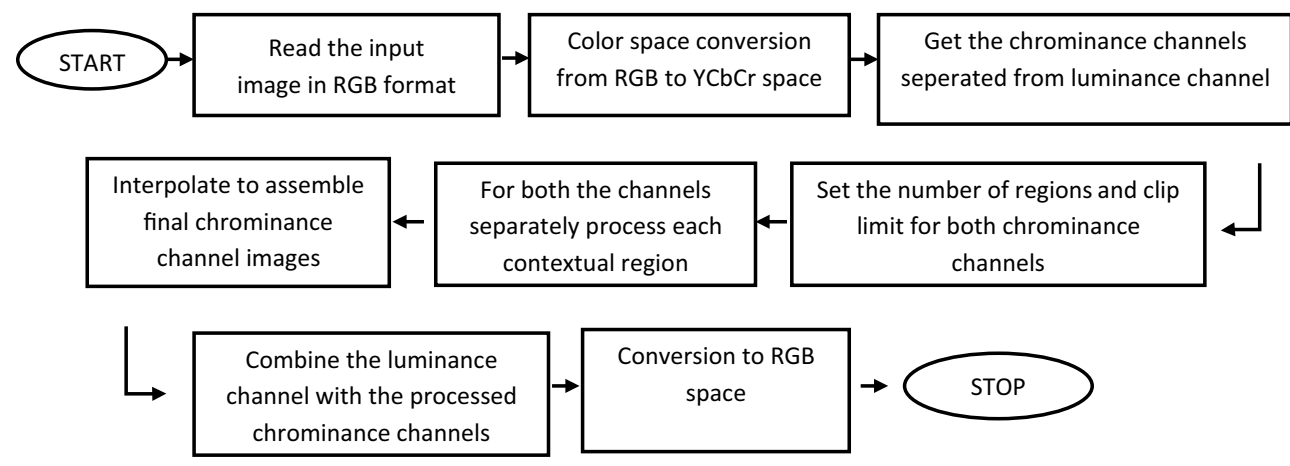

Fig. 8. Flow chart for CLAHE method. 


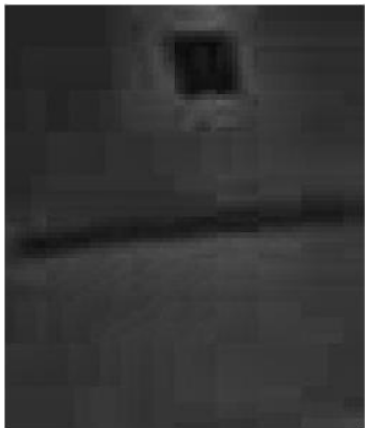

(a)

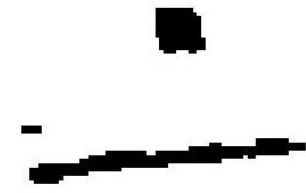

(b)
Fig. 10. In-vitro measurement results; (a) gelatin phantom image, (b) processed vascular (tube) image (small square region denotes reference scaling object).

Table 1. The measurement results about artificial vessel (plastic tube) for depth of $3.5 \mathrm{~mm}$.

\begin{tabular}{lccccc}
\hline \multicolumn{3}{c}{$\lambda=875 \mathrm{~nm}$} & \multicolumn{3}{c}{$\lambda=940 \mathrm{~nm}$} \\
\hline $\mathrm{D}(\mathrm{mm})$ & $\mathrm{D}^{*}(\mathrm{~mm})$ & Error & $\mathrm{D}(\mathrm{mm})$ & $\mathrm{D}^{*}(\mathrm{~mm})$ & Error \\
1 & 1.3 & 0.3 & 1 & 1.9 & 0.9 \\
3 & 2.7 & 0.3 & 3 & 2.9 & 0.1 \\
\hline
\end{tabular}

Note: (D: actual diameter, $\mathrm{D}^{*}$ : calculated diameter).

burned at a depth of $3.5 \mathrm{~mm}$ from the surface. The measurements were repeated for light source with $875 \mathrm{~nm}$ and $940 \mathrm{~nm}$. Figure 10 shows the imaging results for a tube with diameter of $1 \mathrm{~mm}$.
From this image, the diameter of tube was calculated as $1.3 \mathrm{~mm}$ (for $875 \mathrm{~nm}$ ). The measurements were repeated for other tube with diameters of $3 \mathrm{~mm}$ and wavelength of $940 \mathrm{~nm}$, respectively. Consequently, all the obtained results are given in Table 1.

Since, only one plastic tube was used in all measurements (not vascular network), FVD parameter was not calculated.

\subsection{In-vivo imaging results}

In this step, optical system was used for vascular imaging wrist and arm region of 25 years old volunteer female subject. The obtained raw and processed images for wavelength of $875 \mathrm{~nm}$ were shown in Fig. 11. The reason for using wavelength of $875 \mathrm{~nm}$ is that the average error (0.3) (in Table 1) for this wavelength, is less then that of the wavelength $940 \mathrm{~nm}$ (average error: 0.5 ).

From processed image, for selected vascular subregion, vascular diameter was calculated as $1.4 \mathrm{~mm}$. After that, for same subject, we studied on wrist region. In order to mimic actual vascular depth, a previously designed pork gelatin-based thin slab (gelatin phantom) with $3 \mathrm{~mm}$ of width (thickness) was placed on subject's wrist. For this condition, obtained images are shown in Fig. 12. From processed image, vascular diameter was calculated as $1.4 \mathrm{~mm}$.

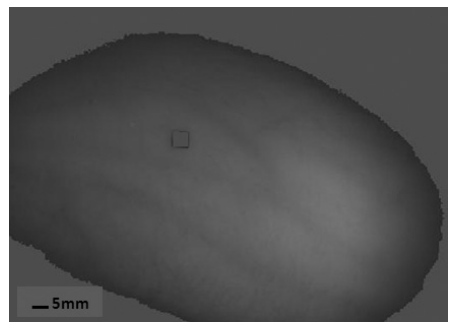

(a)

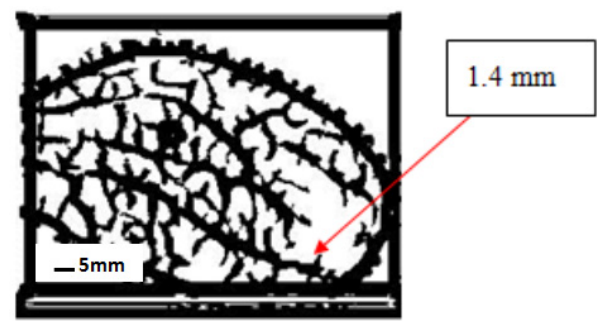

(b)

Fig. 11. In-vivo imaging results (arm region); (a) Raw images, (b) Processed image.

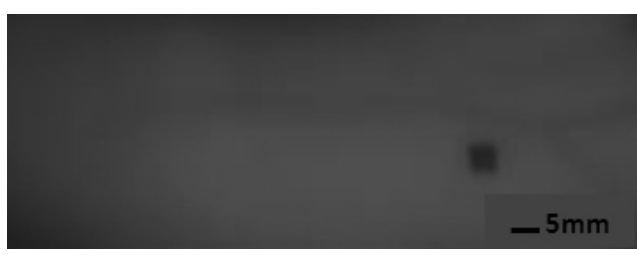

(a)

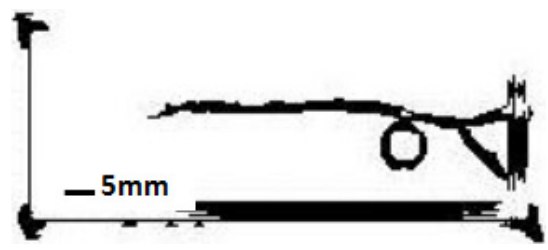

(b)

Fig. 12. The results of in-vivo imaging for wrist region; (a) Raw image, (b) Processed image. 


\section{Conclusions}

In this study, first we designed two types of phantoms, one is liquid based which mimics human blood, and pork gelatin-based solid phantom was used to mimic skin tissue. For both phantoms; absorbing of tissue and blood was mimicked with $0.2 \%$ Indian-ink and $0.1 \%$ intro-lipid solution was used for scattering properties. From designed phantom materials, optical absorption and scattering coefficients were determined via spectrometer and integrating sphere system-based measurements and (IAD) method. In measurement process (or period), despite our designed phantom characteristics not changing, the spectrometer's recordings are made for five trials and its averaged value is taken as a final value so that we obtain statistical reliability about recorded values. According to calculated figures, we observed that liquid phantom's absorption coefficient $\left(\mu_{a}=0.2 \mathrm{~cm}^{-1}\right)$ was in agreement with the data from the literature for real human blood, but reduced scattering coefficient was not $\left(\mu_{s}^{\prime}<<\right.$ literature). In the same way, solid gelatin phantom's reduced scattering coefficient was also smaller than that of literature's figure. We think that these errors are due to the use of integrating sphere's inner walls instead of standard reference material and mixing-heating procedures of gelatin material. On the other hand, absorption coefficient of gelatin phantom was found $\left(\mu_{a}=4.1 \mathrm{~cm}^{-1}\right.$ for $875 \mathrm{~nm}$ ) greater than that of human tissue. We think that this mismatch could be originated from gelatin phantom's specifications (for example: humidity, temperature, density etc.). In future studies: we will plan to make new type gelatin phantoms with different conditions for pork gelatin materials and to optimize fabrication process such as mixing and heating.

Second step of our study consists of NIR optical imaging the designed solid (gelatin) phantoms in which artificial veins (plastic tubes) were inserted to phantom base material for different tube diameters and certain depth level $(3.5 \mathrm{~mm})$. Previous designed liquid phantom which mimics human blood, was injected into tube to get vascular image. From recorded images, diameter and FVD parameter of selected vascular sub-region can be calculated via user-friend interface that consists of our designed image processing and analysis routines. Table 1 shows these calculated and actual figures. From the table, we can see that difference between calculated and actual diameter was $0.3 \mathrm{~mm}$ for $875 \mathrm{~nm}$, however, at $940 \mathrm{~nm}$ two types of difference were obtained, such as $0.1 \mathrm{~mm}$ and $0.9 \mathrm{~mm}$ respectively. We think that these mismatch could be originated from optical system settings (i.e.,: zooming, focusing etc.), and parameter selection for thinning and skelitization image processing steps. In the final step, vascular imaging of volunteer subject was implemented. The calculated vascular diameter (arm region) was found as $1.4 \mathrm{~mm}$. If we considered the error term with $0.3 \mathrm{~mm}$ at artificial vascular measurement for $875 \mathrm{~nm}$, the calculated vascular diameter (in-vivo condition) can be accepted in normal range. In the following, we compare our in-vivo results with those obtained by using very popular method called duplex ultrasound method. In Ref. 27, authors adopted Duplex ultrasound mapping of arm veins to determine diameter of related vein under six different conditions. In clinical practice, duplex mapping appears to have a good predictive diameter value whenever large veins are demonstrated, but its sensitivity (i.e., the ability to detect and accurately measure small veins) is unproven, because veins with diameter $<2 \mathrm{~mm}$, are seldom detected. This result generally is valid for many ultrasound measuring systems. Especially in Ref. 27, the proposed system failed to measure veins with diameter $<1.3 \mathrm{~mm}$. On the other hand, with our measurement system, for in-vivo conditions, it was possible to measure plastic tube with diameter $1 \mathrm{~mm}(\lambda=875 \mathrm{~nm}$, error: $30 \%)$. From this observations, we can say that our proposed method, for in-vivo conditions, provides good results respect to that of ultrasound-based methods that are famous modalities in clinical practice.

Consequently, we showed that the proposed optical system for deep tissue vascular imaging can be used for this aim. However, in future plan, some enhancements about fabrication of solid phantom and optical set up should be required for clinical experiments. On the other hand, since there is no reference FVD values in vivo conditions, this parameter was not discussed here.

\section{Acknowledgment}

This research was supported in-part by Scientific and Technological Research Council of Turkey (TUBITAK), under grand, No:113E771.

\section{References}

1. L. Zhanjun, Z. Yuanwei, W. Xiang, W. Xiaoqiong, M. Rohit, Z. Hongwu, H. Gang, "In vivo repeatedly 
charging near-infrared-emitting mesoporous $\mathrm{SiO} 2 /$ ZnCa2O4:Cr3+ persistent luminescence nanocomposites," Adv. Sci. 1500001(2), 1-6 (2015).

2. L. Zhanjun, Z. Yuanwei, L. Hieu, Z. Richard, E. Ghida, W. Yuzou, H. Gang, "Upconevrting NIR photons for bioimaging," Nanomaterials 5, 21482168 (2015).

3. K. Phipps, A. Modic, M. A. O'Riordan, M. Walsh, "A randomized trial of the vein wiever versus Standard technique for placement of peripherally inserted central catheters (PICCs) in neonates," J. Perinatol. 32(7), 1-4 (2011).

4. D. Yaping, B. Xu, F. Tao, C. Miao, L. Feng, Z. Yan, W. Qiangbin, "Near-infrared photoluminescent Ag2S quantum dots from a single source precursor," J. Am. Chem. Soc. 132(5), 1470-1471 (2010).

5. V. Krasimir, M. Agnieszka, L. H. Andrew, C. Joseph, H. V. Nicolas, D. S. Rob, "Creating gradients of two proteins by differential passive absorption onto a PEG-density gradient," Biometarials 31, 392-397 (2010).

6. H. Guosong, C. L. Jerry, T. R. Joshua, R. Uwe, X. Liming, F. H. Ngan, P. C. John, D. Hongjie, "Multifunctional in vivo vascular imaging using near infrared II fluorescence," Nat. Med. 18, 1841-1846 (2012).

7. H. D. Zeman assignee, Contrast enhancing illuminator, US Patent 5,969,754 Oct 19 (1999).

8. L. Wang, G. Leedham, S. Y. Cho, "Infrared imaging of hand vein patterns for biometric purposes," IET Comput. Vis. 1(3-4), 113-122 (2007).

9. Y. Ding, D. Zhuang, K. Wang, "A study of hand vein recognotion method," Proc. IEEE Int. Conf. Mechatronics Automation, Vol. 4, pp. 2106-2110 (2005).

10. K. Seker, Design of Optical Imaging Based Vascular Evolution Monitoring System for Superficial Tumor Medication, Master thesis, Ege University, Izmir/ TURKEY (2015).

11. T. Myllyla, A. Popov, V. Korhonen, A. Bykov, M. Kinnunen, "Optical sending of a pulsating liquid in a brain-mimicking phantom," Proc. SPIE 8799, 87990X (2013).

12. C. Chen, F. Klampfl, R. Kanawade, M. Riemann, C. Knipfer, F. Stelzle, M. Schmidt, "Recovering the superficial Microvascular pattern via diffuse reflection imaging: Phantom validation," Bio. Med. Eng. Online 14, 87 (2015).

13. K. T. Arun, A. J. Farouc, "Intravascular near-infrared fluorescence molecular imaging of atherosclerosis," $\mathrm{Am}$. J. Nucl. Mol. Imag. 3(3), 217-231 (2013).

14. B. Zhu, E. M. Sevick-Muraca, "A review of performance of near-infrared fluorescence imaging devices used in clinical studies," Br. J. Radiol 88, 20140457 (2015).

15. S. Felix, K. Stefan, J. M. Andreas, Z. Rapheal, M. P. Juan, W. Ursula, W. Martin, "A review on continuous wave functional near-infrared spectroscopy and imaging instrumentation and methodology," Neurolmage 85, 6-27 (2014).

16. J. Simon, Z. Borut, "An innovative approach to near-infrared spectroscopy using a standard mobile device and its clinical application in the real-time visualization of peripheral veins," $\mathbf{1 4}$, 100 (2014).

17. A. H. Holly, "A biomedical device for improve pediatric vascular access seccess," Pediatr. Nurs. 36(5), 259-263 (2010).

18. K. M. Roberto, D. Z. Herbert, H. D. Flavio, K. Rodrigo, R. Eduardo, L. Gunnar, V. Carlos, "Vein imaging: A new method of near infrared imaging, where a processed image is projected onto the skin fort he enhancement of vein treatment," Dermatol. Surg. 32, 1031-1038 (2006).

19. Y. Aycan, A. Lale, S. Bulent, "Hand vein biometry based on geometry and appearance methods," IET Comput. Vis. 5(6), 398-406 (2011).

20. S. W. Maciej, P. P. Alexey, V. B. Alexander, K. Matti, J.-S. Malgorzata, V. T. Valery, "Measurements of fundemental properties of homogeneous tissue phantoms," J. Biomed. Opt. 20(4), 045004 (2015).

21. Oregon Medical Laser Center, Oregon Health \& Science University, Portland, Oregon, USA, http:// omlc.org/ (2016).

22. M. S. Neethu, V. K. Jayasree, "Contrast limited adaptive histogram equalization for qualitative enhancement of myocardial perfusion images," Engineering 5, 326-331 (2013).

23. M. S. Pizer, D. A. John, R. P. John, D. S. Hal, Adaptive Histogram Equalization for Automatic Contrast Enhancement of Medical Images, North Carolina University (1986).

24. N. Fangyan, G. Chao, G. Yongcai, G. Min, "Twodimentional minimum local cross-entropy thresholding based on co-occurance matrix," Comput. Electr. Eng. 37, 757-767 (2011).

25. R. C. Gonzalez, R. E. Woods, Digital Image Processing, Prentice-Hall, New Jersey (2002).

26. S. M. White, C. S. George, B. Choi, Automated computation of functional vascular density using laser speckle imaging in a rodent window chamber model, Microvasc. Res. 82(1), 92-95 (2011).

27. S. Paul, K. Patrick, B. John, Improvement in the visualization of superficial arm veins being evaluated for access and bypass, 17th Annual Meeting of the American Venous Forum, San Diego, Calif (2005). 